\title{
L'obligation de ne pas prêter aide ou assistance au maintien d'une situation créée par la violation d'une norme impérative du droit international général
}

Fecha de recepción: 22 de febrero de 2016

Fecha de aceptación: 10 de mayo de 2016

Doi: 10.12804/revistas.urosario.edu.co/acdi/a.5298

\section{Djamchid Momtaz*}

Résumé: En vue d'empêcher l'affermissement des autorités de fait établies sur un territoire suite à un recours illicite à la force et/ou au mépris du droit des peuples concernés à disposer d'eux-mêmes, le droit international impose des obligations aux Etats tiers. Les traités qu'ils concluent avec les autorités de fait établies sur ces territoires portant sur des questions les concernant doivent garantir l'intérêt des populations soumises à leur juridiction. A défaut, ces Etats doivent s'attendre à ce que les relations conventionnelles établies fassent l'objet de critiques et soient éventuellement dénoncées par les tribunaux tant nationaux qu'internationaux. La même préoccupation est à l'origine de la distinction que le droit international établit entre les différents actes émanant des autorités de fait administrant ces territoires. Ne seront pas entachés de nullité les actes administratifs qu'elles émettent pour préserver les droits civils des personnes qui relèvent de leur juridiction ni ceux qui permettront à la population concernée de

* Professeur à l'Université de Téhéran - Membre de l'Institut de droit international Ancien président de la Commission du droit international.E-mail: djmomtaz@yahoo.com Para citar este artículo: Momtaz, D., "L’obligation de ne pas prêter aide ou assistance au maintien d'une situation créée par la violation d'une norme impérative du droit international général", Anuario Colombiano de Derecho Internacional (ACDI), 2017, 10, pp. 205-219. Doi: 10.12804/revistas.urosario.edu.co/acdi/a.5298 
bénéficier des avantages de la coopération internationale. Distinction difficile à établir et à mettre en œuvre.

Mots clés: Affermissement de l'autorité de fait, recours illicite à la force, droit des peuples à disposer d'eux-mêmes, droit de traiter, intérêt de la population, acte établi par autorité de fait.

The Obligation to not Render Aid or Assistance so as to Maintain a Situation Created by the Breach of a Peremptory Norm of General International Law

Abstract: In order to prevent the consolidation of de facto authorities established in a territory following an unlawful use of force and/or contempt of the rights of the involved peoples to self-determination, international law imposes obligations on third States. Treaties entered into with the de facto authorities established on such territories on matters concerning them must ensure the interests of the people under their jurisdiction. Otherwise, these States must expect that these treaty relations will be criticized and eventually be denounced by national and international courts. Of the same concern is the origin of the distinction that international law establishes between the different acts of the de facto authorities administering these territories. Administrative acts issued to safeguard the civil rights of the people within their jurisdiction will not be null and void unlike those that seek to allow the population to enjoy the benefits of international cooperation. This is a difficult distinction to establish and implement.

Key words: Strengthening de facto authority, unlawful use of force, the right of peoples to self-determination, law of treaties, public interest, act established by de facto authority.

La obligación de no prestar ayuda o asistencia al mantenimiento de una situación creada por la violación de una norma imperativa de derecho internacional general

Resumen: en aras de impedir el fortalecimiento de las autoridades de hecho establecidas sobre un territorio como consecuencia de un uso ilícito de la fuerza y/o en contra del derecho de los pueblos a la autodeterminación, 
el derecho internacional impone unas obligaciones a los terceros Estados. Los tratados que se concluyen con las autoridades de hecho establecidas sobre los territorios portan sobre temas que los intervinientes deben garantizar el interés de las poblaciones sometidas a su jurisdicción. A falta de esto, esos Estados deben esperar a que las relaciones convencionales establecidas sean objeto de críticas y sean eventualmente denunciadas por los tribunales, tanto nacionales como internacionales. La misma preocupación está en el origen de la distinción que el derecho internacional establece entre los diferentes actos emanados de autoridades de hecho que administran dichos territorios. No conllevarán la nulidad de los actos administrativos que ellas profieran para preservar los derechos civiles de personas que xx su jurisdicción, ni aquellos que permiten a la población concernida deben beneficiar de las ventajas de la cooperación internacional. Distinción difícil de establecer y de poner en práctica.

Palabras clave: fortalecimiento de las autoridades de hecho, recurso ilícito al uso de la fuerza, derecho de la autodeterminación de los pueblos, derecho de tratar, interés de la población, acto establecido por la autoridad de hecho.

A obrigação de não prestar ajuda ou assistência à manutenção de uma situação criada pela violação de uma norma imperativa de direito internacional geral

Resumo: Com o objetivo de impedir o fortalecimento das autoridades de facto estabelecidas sobre um território como consequência de um uso ilícito da força e/ou contra o direito dos povos à autodeterminação. $\mathrm{O}$ direito internacional impõe umas obrigações aos terceiros Estados. Os tratados que se concluem com as autoridades de facto estabelecidas sobre os territórios portam sobre temas que os intervenientes devem garantir o interesse das populações submetidas à sua jurisdição. A falta disto, esses Estados devem esperar a que as relações convencionais estabelecidas sejam objeto de críticas e sejam eventualmente denunciados pelos tribunais, tanto nacionais quanto internacionais. A mesma preocupação está na origem da distinção que o direito internacional estabelece entre os diferentes atos emanados de autoridades de facto que administram ditos territórios. Não implicarão a nulidade dos atos administrativos que elas profiram para preservar os direitos civis de pessoas que xx sua jurisdição, nem aqueles que permitem à população concernida devem beneficiar das 
vantagens da cooperação internacional. Distinção difícil de estabelecer e de pôr em prática.

Palavras-chave: fortalecimento das autoridades de facto, recurso ilícito a uso da força, o direito da autodeterminação dos povos, direito de tratar, interesse da população, ato estabelecido pela autoridade de facto.

Les demandes adressées aux Etats par le Conseil de sécurité et l'Assemblée Générale suite à un recours illicite à la force ou au non respect du droit des peuples à disposer d'eux-mêmes ne se limitent pas à une obligation d'abstention de reconnaître les situations créées par ces violations. Dans la plupart des cas, les Etats destinataires sont appelés à se conformer aussi à des obligations positives consistant à encadrer les relations qu'ils entretiendront avec l'Etat auteur de l'acte illicite ou l'entité illégale mise en place par ce dernier. Dans les deux cas, la finalité recherchée est d'empêcher l'affermissement de leur autorité sur les territoires soumis à l'exercice d'une juridiction de fait.

En cas de silence des organisations internationales, les Etats serontils libres d'organiser comme bon leur semble leurs relations avec les Etats responsables et les entités mises en place par ceux-ci? La «Déclaration relative aux principes du droit international touchant les relations amicales et la coopération entre les Etats conformément à la Charte des Nations Unies» ne se prononce pas sur ce point. Elle se contente d'affirmer que «nulle acquisition territoriale obtenue par la menace ou l'emploi de la force ne sera reconnue comme légale». ${ }^{1}$

Lors des travaux préparatoires menés par l'Assemblée Générale en vue de l'élaboration de cette résolution, les Etats qui se sont prononcés

\footnotetext{
1 Résolution 2625 (XXV) adoptée le 24 octobre 1970. Obligation corrélative de l'interdiction de recourir à la menace ou à l'emploi de la force contre l'intégrité territoriale ou l'indépendance politique des Etats, figurant dans la Charte des Nations Unies. Selon la CIJ, les principes énoncés dans la Charte au sujet de l'usage de la force reflètent le droit international coutumier ( $\$ 187$ à 190 de l'arrêt rendu le 27 juin dans l'affaire des activités militaires et paramilitaires au Nicaragua et contre celui-ci Affaire Nicaragua c. Etats-Unis d'Amérique, fond). D'après la Cour, «Cela vaut également pour ce qui en est le corollaire, l'illicéité de toute acquisition de territoire résultant de la menace ou de l'emploi de la force ( $\$ 87$ de l'avis consultatif sur les conséquences juridiques de l'édification d'un mur dans le territoire palestinien occupé, rendu le 9 juillet 2004). Dans les deux affaires citées, la Cour se réfère à la Résolution 2625.
} 
sur le sens et la portée de l'obligation de non reconnaissance étaient d'avis qu'elle ne pouvait avoir de conséquence sur les relations économiques et commerciales entretenues avec l'Etat auteur de l'acte illicite. ${ }^{2}$ L'examen de la jurisprudence récente révèle néanmoins que les Etats ne disposent pas d'une entière liberté d'action dans ce domaine, les intérêts des populations concernées devant être pris en considération, aussi bien lors de la conclusion d'un traité appelé à s'appliquer à un territoire à souveraineté contestée (I) que dans l'appréciation de la légalité des actes émanant des autorités de fait établies sur un tel territoire (II).

\section{Limite à la liberté de conclure un traité portant sur le territoire administré par une autorité de fait}

S'il n'existe pas de règle de droit international coutumier interdisant la conclusion d'un traité devant s'appliquer à un territoire disputé, les Etats n'en sont pas pour autant libres d'en déterminer le contenu. Les dispositions d'un tel traité ne sauraient en effet méconnaître les obligations erga omnes qu'ils sont tenus de respecter, en l'occurrence le respect du droit des peuples à disposer d'eux-mêmes et de son corollaire la souveraineté permanente sur les ressources naturelles tant minérales qu'agricoles que le territoire contesté recèle.

\section{Les Etats sont en droit de traiter avec l'autorité de fait}

Suite à l'annexion par l'Indonésie du Timor oriental, territoire non autonome administré par le Portugal, on s'est posé la question de savoir si ce dernier gardait toujours le monopole d'établir avec les Etats tiers des relations conventionnelles portant sur ce territoire. Le Portugal demandait alors à la Cour internationale de justicie (CIJ) de dire et de juger que l'Australie avait violé ses obligations internationales du fait de la conclusion avec l'Indonésie d'un accord sur la délimitation du plateau continental du Timor oriental.

\footnotetext{
2 Hans Blix Contemporary Aspects of Recognition Recueil des cours de l'Académie de droit international, Tome 130 (1970) pp. 664-665. D’après l'auteur, qui a représenté la Suède lors des négociations ayant abouti à l'adoption de la Résolution 2625, «If States were asked not to recognize or consider as legal that which was illegal, there would be no great difficulty. The difficulties lay in the fact that the duty of «non recognition» might be argued by some to constitute a bar against trade arrangements, normal communication and practical contacts of any kind with the accused State. It would be hardly realistic for any State to make so broad an understanding» (UN Doc. A/AC.125/SR.86).
} 
Selon l'Australie, à défaut d'une décision du Conseil de sécurité, «Il n'existe aucun critère sur la base duquel une obligation automatique de non reconnaissance pourrait être invoquée». De plus, «Il est irréaliste et injuste d'imposer à des Etats tiers les inconvénients très considérables de la non reconnaissance, tant sur le plan économique que du point de vue de leurs relations avec l'Etat visé. ${ }^{3}$

La Cour internationale de justice semble épouser cette thèse puisqu'elle est d'avis que les résolutions adoptées par le Conseil de sécurité n'imposent pas aux Etats l'obligation de ne reconnaître à l'Indonésie aucune autorité à l'égard du territoire et de ne traiter les questions qui le concernent qu'avec le Portugal. ${ }^{4}$ La Cour semble se fonder sur la situation de fait qui prévaut au Timor, soumis à l'autorité de l'Indonésie, pour parvenir à une telle conclusion. Il n'en reste pas moins que si les conditions étaient réunies pour que la Cour puisse dépasser le stade des exceptions préliminaires, elle se serait sans doute préoccupée de la licéité de l'accord conclu, accord qui méconnaît les intérêts du Timor oriental dans l'exploitation des ressources naturelles de son plateau continental au mépris de sont droit à l'autodétermination, qualifié par la Cour dans cette même affaire de droit opposable erga omnes. ${ }^{5}$ La jurisprudence ultérieure des juridictions internationales révèle qu'il existe désormais une règle bien établie obligeant les Etats exerçant leur autorité sur un territoire disputé à exploiter ses ressources naturelles dans l'intérêt de la population concernée.

\section{Le traité conclu doit garantir l'intérêt des habitants du territoire}

L'accord qui vient d'être conclu entre la Turquie auto proclamée et la République turque de Chypre du Nord pour la délimitation de leur plateau continental ne pourra être considéré a priori comme n'ayant pas pris en

\footnotetext{
3 Plaidoirie de James Crawford, conseil de l'Australie devant la CIj; CR 1995/9 \ 88 et 92.

4 Timor oriental (Portugal c. Australie) arrêt rendu par la CIJ le 30 juin 1995. La Cour rejette l'argument du Portugal selon lequel les résolutions adoptées par le Conseil de sécurité suite à l'annexion su Timor oriental imposent aux Etats l'obligation de ne traiter, en ce qui concerne ce territoire, qu'avec le Portugal. La Cour «n'est pas convaincue que lesdites résolutions soient allées aussi loin» \31 de l'arrêt.

5 Selon la Cour, «Il n'y a rien à redire à l'affirmation du Portugal selon laquelle le droit des peuples à disposer d'eux-mêmes, tel qu'il s'est développé à partir de la Charte et de la pratique de l'Organisation des Nations Unies, est un droit opposable erga omnes» \29 de l'arrêt.
} 
compte l'intérêt de la population établie sur le territoire de cette entité sécessionniste. C'est la raison pour laquelle la République de Chypre se fonde, pour la condamner, plutôt sur la résolution du Conseil de sécurité demandant aux Etats de «ne pas encourager ni aider d'aucune manière l'entité sécessionniste ${ }^{6}$. Il ne fait en effet aucun doute que l'exploitation des ressources du territoire de cette entité ne se fera pas aux dépens de la population qui y est établie. La même remarque s'impose pour ce qui est de l'importation par les pays de l'Union européenne de marchandises et produits agricoles provenant de ce même territoire s'ils ne sont pas accompagnés de certificats délivrés par les autorités de la République de Chypre. $^{7}$ Les relations économiques qui seraient établies sans l'agrément de cette derniere seraient de nature à affermir l'autorité de l'entité sécessionniste de Chypre du Nord. ${ }^{8}$

Il en va de même de l'interdiction d'importation par les Etats membres de l'Union européenne de produits originaires des colonies de peuplement israéliennes créées sur les territoires palestiniens occupés bénéficiant des traitements tarifaires préférentiels prévus par l'accord d'association Israël-Union européenne. En effet, cette importation serait incontestablement de nature à consolider l'occupation illégale de ces territoires par Israël et se ferait aussi aux dépens de la population palestinienne. ${ }^{9}$ Le récent arrêt

6 Rés. 550 du 11 mars 1981. Cet accord a été dénoncé par la République de Chypre (lettre datée du 20 mars 2014 Doc. NU A/68/883). Accord ratifié par la Turquie le 21 septembre 2011 et publié par le Bulletin du droit de la mer Cf. EJIL talk 26 mai 2014.

7 Affaire C-432/92 Décision à titre préjudiciel sollicitée par la High Court of Justice (Queen's Bench Division) dans l'affaire The Queen and Minister of Agriculture, Fisheries and Food, ex parte / SP Anastasiou (Pissouri) Ldt e.q. (\$ 67) Cf. Theodore Christakis L'obligation de non reconnaissance des situations créées par le recours illicite à la force ou d'autres actes enfreignant des règles fondamentales in The Fundamental Rules of International Legal Order, Jus cogens and obligations erga omnes Tomuschat C. et Thouvenin J. M. (Eds.), Martinus Nijhoff Publishers, 2006, pp. 155-156.

8 Conséquences juridiques pour les Etats de la présence continue de l'Afrique du Sud en Namibie (Sud-Ouest africain) nonobstant la Résolution 276 (1970) du Conseil de sécurité. D'après la CIJ dans son avis consultatif du 21 juin 1971, «Les restrictions qui impliquent la non reconnaissance de la présence de l'Afrique du Sud en Namibie et les dispositions expresses du $\ 5$ de la Résolution 276 (1970) imposent aux Etats membres l'obligation de ne pas entretenir avec l'Afrique du Sud agissant au nom de la Namibie ou en ce qui la concerne des rapports ou des relations de caractère économique ou autre qui seraient de nature à affermir l'autorité de l'Afrique du Sud dans le territoire» $\$ 124$ de l'avis.

9 C. Hauswaldt Problems Under the EC-Israel Association Agreement: the Export of Goods produced in the West Bank and the Gaza Strip Under the EC-Israel Association Agreement (2003) 
de la Cour de justice de l'Union européenne dans l'affaire Brita vient de préciser une nouvelle fois que cet accord n'est applicable qu'au territoire de l'Etat d'Israël à l'exclusion des territoires palestiniens occupés. ${ }^{10}{ }^{S}$ 'il est vrai que l'exploitation des fermes dans les colonies de peuplement contribue à créer de l'emploi pour les Palestiniens, il n'en reste pas moins que les grands bénéficiaires en sont les colons eux-mêmes dont l'implantation sur les territoires palestiniens occupés a été à maintes fois condamnée par le Conseil de sécurité comme contraire au droit international humanitaire. ${ }^{11}$

C'est sans doute l'avis du conseiller juridique des Nations Unies qui met le mieux en exergue la volonté des Etats de s'opposer à l'exploitation des ressources des territoires disputés entreprise aux dépens de leurs habitants. Interrogé sur la légalité des concessions accordées en octobre 2001 par le Maroc pour l'exploitation du pétrole du Sahara occidental, il fit remarquer que l'Assemblée Générale a toujours favorisé l'exploitation des ressources des territoires non autonomes lorsqu'elle est entreprise au bénéfice des peuples concernés, en leur nom ou en consultation avec leurs représentants. Ce n'est que dans ces conditions que la mise en valeur de ces ressources serait compatible avec les obligations que la Charte impose aux Etats qui administrent ces territoires, dont le respect de la souveraineté sur leurs ressources naturelles. ${ }^{12} \mathrm{Il}$ en va évidemment de même pour ce qui est des ressources halieutiques et des produits agricoles du Sahara

14 EJIL, p. 591. Le 12 mars 1998, la Cour de justice des Communautés européennes avait déclaré qu'Israël était suspecté d'avoir violé l'accord d'association en exportant dans l'espace européen des produits originaires des territoires palestiniens occupés au mépris du statut international de ces territoires.

10 Arrêt du Tribunal (quatrième Chambre) du 25 février 2010 Firma Brita Gmbh contre Haupzollamt Hambourd-Hafin (Arrêt C-386 / 08) Cf. Matteo Fornari La Cour de justice de l'Union européenne se prononce sur l'importation de produits fabriqués dans les territoires palestiniens occupés: verre demi plein ou verre demi vide in International Courts and the Development of International Law: Essays in Honour of Tullio Treves / Nerina Boschiero (et al.) Asser Press, 2013, pp. 897 et s. Cf. Décision de la Commission européenne du 11 novembre 2015 C (2015) 7834 concernant l'étiquetage par l'Union européenne des produits en provenance des territoires palestiniens occupés.

11 Art. 49 de la Convention de Genève du 12 août 1949 relatif à la protection des personnes civiles en temps de guerre - Résolution 446 du 22 mars 1979 qui considère que la politique et les pratiques israéliennes consistant à établir des colonies de peuplement dans les territoires palestiniens et autres territoires arabes occupés depuis 1967 n'ont aucune validité en droit.

12 Avis du sous-secrétaire général des Nations Unies aux affaires juridiques Hans Corell au Président du Conseil de sécurité S/2002/61 - 12 octobre 2002 \ 24. 
occidental, exportés vers les Etats de l'Union européenne conformément aux accords conclus avec le Maroc.

Le Front Polisario, agissant au nom du peuple sahraoui, demandait à la Cour de justice de l'Union européenne l'annulation de l'une des décisions du Conseil de l'Union relative à ces transferts. ${ }^{13}$ Selon la Cour, qui fit droit à ce recours, le Conseil, à défaut du Maroc, «était tenu de garantir que l'exploitation des ressources du Sahara ne se fasse pas au détriment de ses habitants ou qu'elle ne porte pas atteinte à leurs droits fondamentaux ». ${ }^{14}$ Or, selon la Cour, il s'est avéré que l'exploitation était entreprise «au mépris des intérêts et de la volonté du peuple de ce territoire et qu'elle contreviendrait aux principes du droit international». ${ }^{15}$

\section{L'aménagement de l'obligation de non reconnaissance des actes émis par l'autorité de fait}

On ne saurait invoquer l'intérêt de la population d'un territoire pour justifier la conformité de l'exportation des ressources originaires de ces territoires avec le droit international et le méconnaitre lors de la mise en œuvre de l'obligation de non reconnaissance des actes de l'autorité de fait administrant ce même territoire. Dans les deux cas, ce qui est recherché est d'éviter que l'Etat qui a violé la norme impérative du droit international ne puisse consolider la situation qui en résulterait à son avantage et au détriment de la population concernée. La sanction de l'Etat responsable ne devrait donc pas avoir pour conséquence de compromettre les droits fondamentaux de ladite population, situation qu'une application rigide de l'obligation de non reconnaissance des actes émis par l'autorité de fait risquerait d'engendrer. Son aménagement dans l'intérêt de la population s'impose donc. La CIJ l'a préconisé dans l'avis qu'elle a émis dans l'affaire

13 Décision 2012/497/uE du Conseil en date du 8 mars 2012 concernant la conclusion de l'accord, sous forme d'échange de lettres entre l'Union européenne et le Royaume du Maroc relative aux mesures de libération réciproque en matière de produits agricoles transformés de poissons et de produits de la pêche.

14 Arrêt du Tribunal (huitième Chambre) du 10 décembre 2015 dans l'affaire T-512/12 Front populaire pour la libération de la Sanguia-el-Hamra et du Rio de Oro (Front Polisario) c. Conseil de l'Union européenne \246-247.

15 \222 de l'arrêt de la Cour de justice de l'Union européenne se référant à l'argument du sous-secrétaire général des Nations Unies Cf. op. cit., note (12) Enrico Milano The New Fisheries Partnership Agreement Between the European Union and the Kingdom of Morocco: Fishing to Far South? 22 Annuario Español de Derecho Internacional (2006), pp. 413-457. 
de la Namibie. Certes, l'aménagement ne doit néanmoins pas aboutir à renforcer la position de l'autorité de fait.

\section{L'aménagement dans l'intérêt de la population du territoire administré par une autorité de fait}

A la suite du retrait du mandat de l'Afrique du Sud sur la Namibie décidé par le Conseil de sécurité, ${ }^{16}$ la CIJ déclarait, à l'instar du Conseil de sécurité, dans l'avis émis à sa demande, que «Toutes les mesures prises par le gouvernement Sud-africain au nom de la Namibie ou en ce qui la concerne après la cessation du Mandat sont illégales et invalides $\gg .{ }^{17}$ Néanmoins, dans l'intérêt du peuple namibien cette nullité ne saurait s'étendre aux avantages qu'il peut tirer de la «coopération internationale» et s'appliquer aux actes administratifs quotidiens.

La coopération internationale peut embrasser des domaines extrêmement variés. Or, la Cour ne précise pas ce que cette expression peut ici englober. On peut songer à l'interdépendance économique de la Namibie à l'égard de l'A frique du Sud, l'imbrication de leurs appareils administratifs étant telle qu'on ne peut l'interrompre brutalement sans porter tort aux intérêts du peuple namibien. De même, en cas de famine ou d'une épidémie consécutive à une catastrophe naturelle, la coopération intergouvernementale s'imposera de toute évidence. ${ }^{18}$ Pour ce qui est de l'exception des actes administratifs quotidiens dont la légalité ne serait pas remise en cause, la Cour cite à titre d'exemple les certificats de naissance, de mort et de mariage. ${ }^{19}$ La Cour s'est sans doute inspirée de la jurisprudence de la Cour suprême des Etats-Unis relative à la validité des actes accomplis par les autorités confédérées du Sud pendant la guerre de sécession dans des domaines similaires. ${ }^{20}$ Cette jurisprudence ne semble pas avoir été abandonnée par les Etats-Unis, les actes privés établis par une entité non

\footnotetext{
16 Rés. 276 - 30 janvier 1970 Cf. note (8), op. cit.

17 \ 125 de l'avis de la CIJ dans l'affaire de Namibie, op. cit., note (8).

18 Opinion individuelle de M. Dillard jointe à l'avis de la CIJ dans l'affaire de Namibie CIJ Recueil 1971, p. 166.

$19 \int 125$ op. cit., note (8).

20 Cf. entre autres Affaire Texas c. White, 74 us 227: 7 Wall, 700 (1868). Dans sa jurisprudence relative à la validité des actes accomplis par les autorités confédérées du Sud pendant la guerre de sécession, la Cour suprême a reconnu dans des limites très strictes une certaine validité aux actes administratifs et aux jugements des instances confédérées
} 
étatique ou un régime non reconnu par les Etats-Unis n'étant pas considérés comme entachés de nullité. ${ }^{21}$

Il semble que le droit international reconnaisse désormais la légitimité de certains arrangements et transactions juridiques effectués par les organes d'un Etat établi sur un territoire dont la souveraineté est contestée. Telle est la conclusion à laquelle est parvenue la Cour européenne des droits de l'homme dans le jugement qu'elle a rendu sur la légalité de certains actes émis par la République turque autoproclamée de Chypre du Nord. A l'appui, la Cour se réfère à la jurisprudence de la CIJ et cite à titre d'exemples des actes bénéficiant d'un tel traitement, notamment ceux identifiés par la CIJ, à savoir les actes relatifs à l'état civil des personnes comme les certificats de naissance, de mariage et de décès dont le refus de reconnaissance équivaudrait à dépouiller les personnes établies sur ce territoire de tous leurs droits. ${ }^{22}$

\section{L'aménagement ne doit pas renforcer la position de l'autorité de fait}

La Cour européenne des droits de l'homme, dans une autre affaire concernant l'activité des organes de la République turque de Chypre du Nord, se fonde une nouvelle fois sur la jurisprudence de la CIJ pour reconnaître la validité de certaines décisions des tribunaux établis par l'entité sécessionniste. ${ }^{23}$ L'affaire portait sur la violation par cette entité de certaines dispositions de la Convention européenne des droits de l'homme que la Grèce imputait à la Turquie. Selon la Cour, les victimes ou leurs ayants droit doivent auparavant épuiser les voies de recours internes mises à leur disposition par la République turque de Chypre du Nord. La Cour estime

pour autant qu'elles ne fussent pas incompatibles avec l'autorité du gouvernement fédéral et ne portassent pas atteinte aux droits constitutionnels des citoyens américains.

21 us Restatement of the Law (Third) The Foreign Relations Laws of the Us Vol. I Minnesota: St Paul 1985 \$205.

22 Affaire Loizidon c.Turquie (Requête n 15318/89) Arrêt du 18 décembre 1996 \ 45. Cf. Paul Tavernier Le droit international dans la jurisprudence de la Cour européenne des droits de l'homme: l'apport des arrêts Loizidon c. Turquie in Du droit interne au droit international, le facteur religieux et l'exigence des droits de l'homme Mélanges Raymond Goy Publications de l'Université de Rouen 1998 p. 416 et s.

23 Affaire Chypre c. Turquie (Requête n 25781/94) Arrêt du 10 mai 2001 Paul Tavernier En marge de l'arrêt Chypre contre Turquie: l'affaire chypriote devant la Cour de Strasbourg Revue trimestrielle des droits de l'homme pp. 807-835. 
une nouvelle fois que la situation qui perdure dans le nord de Chypre suite à l'invasion de l'île par la Turquie se caractérise par l'exercice de l'autorité de fait par la République turque de Chypre du Nord. Pour se justifier, elle se réfère à sa jurisprudence antérieure ainsi qu'à l'avis consultatif de la CIJ dans l'affaire de Namibie, reconnaissant la légitimité de certains arrangements et transactions juridiques émanant de cette autorité. ${ }^{24} \mathrm{D}$ 'après la Cour, et dans la mesure où la République de Chypre ne peut exécuter dans la partie nord de l'île les obligations que la Convention européenne lui impose, toute autre conclusion conduirait à un vide juridique regrettable dans le système de protection des droits de l'homme. ${ }^{25}$ La faculté de demander la protection des tribunaux établis par l'autorité de fait serait donc dans l'intérêt des habitants grecs de cette partie de l'île. ${ }^{26}$ La Cour semble néanmoins consciente que l'interprétation extensive qu'elle donne de l'avis de la CIJ peut avoir pour conséquence de consolider la position de la République turque de Chypre du Nord. Pour l'éviter, elle prend soin de préciser que les recours que l'entité sécessionniste offre «peuvent passer» pour des recours internes. Formule qui sous-entend que ces recours ne doivent pas être considérés comme des recours internes en tant que tels. Elle note par ailleurs que ces recours doivent être appréciés au cas par cas. ${ }^{27}$

En dépit de ces précautions, l'arrêt rendu par la Cour fera l'objet de très vives critiques de la part des juges minoritaires. Il a été soutenu que cette décision aurait pour conséquence de reconnaître dans certains cas la validité des décisions des tribunaux établis par l'autorité de fait. En statuant ainsi, la Cour n'a pas tenu compte de la décision du Conseil de sécurité appelant les Etats à «ne pas encourager ni aider l'entité sécessionniste». ${ }^{28}$ De surcroît, une telle décision n’était absolument pas nécessaire. ${ }^{29}$ En effet, il a été rappelé que la pratique internationale révèle que l'épuisement des recours internes ne doit jamais être une condition sine qua non à une solution internationale, surtout s'il s'avère être futile. ${ }^{30} \mathrm{Au}$

\footnotetext{
24 \ 90 de l'arrêt, op. cit., note (23).

25 Ibid., $\int 78$ et 91 .

26 Ibid., $\int 98$.

27 Ibid., $\int 102$.

28 Rés. 550, op. cit., note (6).

29 Opinion en partie dissidente de Madame la juge Palm jointe à l'arrêt p. 106.

30 Opinion en partie dissidente de M. le juge Marcus-Helmans jointe à l'arrêt pp. 125 et s.
} 
cas où il est établi que les voies de recours internes sont manifestement vaines, leur épuisement n'a pas été exigé comme une condition préalable à l'exercice de la protection diplomatique. ${ }^{31}$

En définitive, dans ce cas, l'aménagement de la règle de non reconnaissance par la Commission européenne des droits de l'homme concerne des «actes de jure imperii ou consolidateurs d'un titre ou d'une autorité illégale». ${ }^{32}$

Doit-on dès lors estimer que les arrêts qui seraient rendus par les tribunaux de la République turque de Chypre du Nord ne pourront être considérés comme des actes concernant les «droits des particuliers» ? Des doutes peuvent être exprimés à ce sujet.

La jurisprudence internationale semble confirmer la cristallisation d'une norme obligeant les Etats à ne pas prêter aide et assistance au maintien d'une situation créée par la violation d'une norme impérative du droit international général. Une telle obligation se trouve d'ailleurs incluse dans le projet d'articles sur la responsabilité des Etats pour fait illicite adopté par la CDI qui peut désormais être considéré comme codifiant le droit international en la matière. ${ }^{33}$ On s'accorde aussi sur le fait que reconnaître la légalité des actes émis par les autorités établies sur un territoire suite à cette violation contribuerait au maintien de la situation créée. Néanmoins, en vue de tenir compte de l'intérêt des habitants de ce territoire, la nécessité d'exclure certains actes dont ils sont bénéficiaires s'est imposée. Le critère retenu par la CIJ dans l'affaire de Namibie s'est avéré soulever des difficultés en pratique. En effet, reconnaître la légalité d'un acte pourrait légitimer l'activité de l'organe auteur de la décision et, en définitive, de l'autorité de fait établie sur le territoire. Il en sera ainsi si l'organe en question occupe une place privilégiée dans la hiérarchie institutionnelle de cette autorité. Dans ces cas, la reconnaissance de la légalité de l'acte

31 Projet d'articles sur la protection diplomatique et commentaires y relatifs (2006). Texte adopté par la Commission du droit international et soumis à l'Assemblée Générale dans le cadre de son rapport A/61/10 Cf. art. 15 «Exceptions à la règle de l'épuisement des recours internes» et le commentaire de cette disposition.

32 Theodore Christakis, op. cit., note (7) p. 162.

33 Projet d'articles de la CDI sur la responsabilité de l'Etat pour fait internationalement illicite (2001), texte adopté par la CDI et soumis à l'Assemblée Générale dans le cadre de son rapport A/56/10 et Corr. 1. Selon l'article 41 al. 2 de ce projet: Aucun Etat ne doit reconnaître comme licite une situation créée par une violation grave d'une obligation découlant de normes impératives du droit international, ni prêter aide ou assistance au maintien de cette situation. 
doit être entourée de précaution et comporter une clause évitant qu'elle ne puisse être interprétée dans ce sens. Il appartiendra à la jurisprudence, tant interne qu'internationale, de trouver la formule adéquate et éviter que la légalisation de l'acte n'aboutisse à consolider la position de l'autorité de fait et assurer son effectivité.

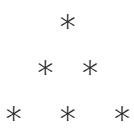

Plus que le corollaire de l'obligation de non reconnaissance des mutations territoriales par la force, celle de ne pas prêter aide ou assistance à leur maintien apparait en effet être son complément. Elle sera de nature à transformer la fiction du statu quo territorial qu'est la non reconnaissance en une réalité. Ainsi, le respect de l'obligation de non assistance serait d'une efficacité accrue. Elle pourra empêcher que le temps n'efface les conséquences de l'illégalité et que l'effectivité ne triomphe aux dépens des normes impératives du droit international général.

\section{Références}

\section{Ouvrages}

Fornari, M., La Cour de Justice de l'Union Européenne se prononce sur l'importation de produits fabriqués dans les territoires palestiniens occupés: verre demi plein ou verre demi vide? International Courts and the Development of International Law. TMC Asser Press, 2013, 897-910.

Goy, M. R., Du droit interne au droit international, le facteur religieux et l'exigence des droits de l'homme, Publications de l'Université de Rouen 1998.

Hans, B., Contemporary Aspects of Recognition, Recueil des cours de l'Académie de droit international de la Haye, Tome 130, 1970.

Theodore, C., L'obligation de non reconnaissance des situations créées par le recours illicite à la force ou d'autres actes enfreignant des règles fondamentales in The Fundamental Rules of International Legal Order, Jus cogens and obligations erga omnes. Tomuschat C. \& Thouvenin J. M. (Eds.). Martinus Nijhoff Publishers, 2006. 


\section{Revues}

Hauswaldt, C., Problems Under the EC-Israel Association Agreement: the Export of Goods produced in the West Bank and the Gaza Strip Under the EC-Israel Association Agreement, EJIL, 2003, 14, 3.

Milano, E., The New Fisheries Partnership Agreement between the European Union and the Kingdom of Morocco: Fishing to Far South? Annuario Español de Derecho Internacional, 2007, 22, pp. 413-457.

Tavernier, P., En marge de l'arrêt Chypre contre la Turquie: l'affaire chypriote et les droits de l'homme devant la Cour de Strasbourg (obs/s. Cour eur. dr. h., Gde ch., Chypre c. Turquie, 10 mai 2001) in Revue trimestrielle des droits de l'homme, 2002, 52, pp. 807-835.

\section{Jurisprudence}

\section{Cour Internationale de Justice}

\section{Avis Consultatifs}

Conséquences juridiques pour les Etats de la présence continue de l'Afrique du Sud en Namibie (Sud-Ouest africain) nonobstant la résolution 276 (1970) du Conseil de sécurité, avis consultatif, CIJ Recueil 1971.

\section{Décisions}

Timor oriental (Portugal c. Australie), arrêt, CIJ Recueil 1995.

\section{Arbitrages}

\section{Documents}

Convention de Genève du 12 août 1949, Art. 49.

Judgment of the General Court (Eighth Chamber) of 10 December 2015. Case T-512/12.

un Doc. A/AC.125/SR.86. 


\section{Traités}

L'affaire T-512/12 Front populaire pour la libération de la Sanguia-elHamra et du Rio de Oro (Front Polisario) c. Conseil de l'Union européenne $\$ 246-247$. 